\title{
A rare case of lobular breast cancer metastasising to large bowel
}

\author{
Waka Yanagisawa $^{1}$, Sandra Krishnan ${ }^{2}$, and Adrian Fernandez ${ }^{2}$ \\ ${ }^{1}$ Affiliation not available \\ ${ }^{2}$ Bega - South East Regional Hospital
}

January 30, 2021

\begin{abstract}
Lobular breast cancer is rarely known to metastasise to the gastrointestinal tract. This article explores a case of a patient in remission from lobular breast cancer, who presented with bowel obstruction secondary to metastatic lobular cancer.
\end{abstract}

\section{Introduction}

Breast cancer is the most common malignancy in women, most frequent in the fourth and fifth decades of life and comprises $27 \%$ of all cancers ${ }^{2}$. Since the introduction of the national breast screening programme and earlier detection, only $7 \%$ of breast malignancies have metastasised at the time of presentation ${ }^{2}$. Breast cancer most commonly metastasise to lungs, bones, liver and brain. On rare occasions, invasive lobular carcinoma (ILC) has been demonstrated to metastasise to the gastrointestinal tract. We describe a case of a patient in remission from lobular breast cancer who presented with metastatic lobular breast carcinoma requiring an extended right hemicolectomy. As there is still a lot to be learnt in regard to metastatic spread of the disease,

this case report raises the discussion regarding benefits of investigating patients with known invasive lobular breast cancer through screening endoscopy, for potential gastrointestinal metastases.

\section{Case Report}

A 78-year-old female presented with a one-month history of colicky abdominal pain, vomiting and unintentional weight loss. Her past medical history included ischemic heart disease on clopidogrel and previous history of deep vein thrombosis. She recently returned a positive stool occult blood test, awaiting colonoscopy. Her past surgical history included a hysterectomy, cholecystectomy, appendectomy and notably, a right lobular breast cancer for which she had undergone a wide local excision and sentinel lymph node biopsy in 2018. Her breast tumour was a Stage IIB, 41mm grade 2 lobular type breast cancer (ER/PR positive, HER 2 negative, Ki-67 of 4\%) with one third of the biopsied lymph nodes positive. She received adjuvant radiotherapy over 6 weeks and hormonal therapy letrozole which was discontinued after 7 months due to arthralgia severely impacting her quality of life. Tamoxifen was deemed unsuitable due to associated risk of thromboembolism and her previous history of deep vein thrombosis. She continued regular outpatient surveillance and was in remission, confirmed by a normal breast ultrasound and mammogram a year prior to presentation.

An Abdominal Computed Tomography on presentation demonstrated concentric wall thickening and narrowing of the distal transverse colon with dilatation of the proximal transverse colon and ascending colon, concerning for a neoplastic lesion (Figures 1,2). Subsequent investigation with a colonoscopy revealed a circumferential lesion in the proximal descending colon, not typical in appearance for an adenocarcinoma. It was difficult to negotiate past the tumour and biopsies were taken. This revealed features suggestive of a sessile serrated adenoma. A semi-urgent laparotomy was performed in view of her progressive abdominal 
symptoms and found the offending, thickened segment to involve the distal transverse colon and splenic flexure. An extended right hemicolectomy was performed, and her surgery was routine. Histopathology revealed a circumferential, firm, ill-defined partially ulcerated lesion measuring $25 \times 20 \mathrm{~mm}$ that was demonstrated to be a metastatic, grade 2 lobular carcinoma deposit. The tumour was centred primarily in the muscularis with extension to the mucosa and subserosa. Lymphovascular was present and three separate extranodal deposits of metastatic lobular carcinoma were seen in the mesenteric tissue. The immunohistochemistry demonstrated ER and PR positive status, HER2 ISH negative and a Ki-67 of $18 \%$ in keeping with the previous lobular breast carcinoma histopathology.

She recovered well post-operatively, requiring a short period of rehabilitation. She was re-referred to the oncology service where she was reviewed one month post-operatively. She was recommenced on letrozole to reduce the risk of further recurrence and associated issues including ascites and pleural effusion. She was not a candidate for chemotherapy given her frailty. Unfortunately, prior to further follow-up, she passed away, two months post-operatively with increasing anorexia and fatigue.

\section{Discussion}

ILC accounts for $10 \%$ of all breast cancers, and is the second most common breast carcinoma ${ }^{1,3}$. The incidence is increasing in postmenopausal women, possibly secondary to the use of hormone replacement therapy in this age group ${ }^{3}$. Breast cancer frequently metastasises to skeletal and pulmonary systems however rarely to the gastrointestinal tract. However lobular breast cancer more frequently metastasise to the gastrointestinal tract ${ }^{4}$. In a study of 2605 cases of breast cancer with metastases; 359 lobular and 2246 with ductal carcinoma, $4.5 \%$ in ILC metastasised to the gastrointestinal tract compared with $0.2 \%$ in the ductal carcinoma group ${ }^{5}$. Gastrointestinal tract involvement with metastatic ILC are thought to most commonly involve stomach and small bowel, then followed by colon and rectum ${ }^{3}$. According to available literature, $60 \%$ metastasises to the stomach, $12 \%$ to the oesophagus, $11 \%$ to the colon, $8 \%$ to the small intestine and $7 \%$ to the rectum ${ }^{6}$. Due to high expression of steroid hormone receptors in lobular breast cancer, adjuvant endocrine treatment is the preferred treatment for $\mathrm{ILC}^{7}$. Patients are characterised by poor prognostic profile and few survive longer than two years following metastasis ${ }^{1,8}$. The reasons for this mechanism of spread are unclear however may possibly include the histopathology of ILC. ILC is characterised by small cells that infiltrate the breast stroma in a single-file 'Indian-file' pattern which does not destroy anatomical structure nor result in a reactive connective tissue response (Figure 3), and can therefore fail to distinctly form a mass in the breast which can be difficult to identify early ${ }^{3}$. This may account for the higher distant metastatic rate in ILCs ${ }^{9}$. Other reasons for metastasis of lobular breast cancer may be explained by E-cadherin which is crucial in the maintenance of epithelial cell polarisation. E-cadherin is a calcium dependent epithelial cell adhesion molecule when mutated or lost can be associated with metastases as it acts as an invasion suppressor ${ }^{10}$. Deficiency of this molecule is responsible for cancer metastasis due to loss of cell to cell adhesion, with increased cell motility causing spread in blood and lymphatics ${ }^{7}$. In lobular breast cancer, E-cadherin loss has been observed in most cases but not observed in invasive ductal cancers. In some studies, E-cadherin has been used to be reliably used as a marker to differentiate between IDC and ILC ${ }^{(11)}$. This may account for its aggressive behaviour compared with IDC. ${ }^{10}$. However poor prognostic factors include age over 70 years, and degree of nodal involvement, which are associated with significant increased risk of relapse and death ${ }^{12}$, which in this case may have increased her risk of poorer outcome. The rarity of the disease and the uncertainty of the nature of ILC makes this an intriguing field, requiring further research and thoughtful consideration in order to most appropriately manage patients. Patients diagnosed with breast ILC and particularly those above 70 years of age, should be carefully monitored for any evidence of potential gastrointestinal metastases through imaging or screening colonoscopy.

\section{Conclusion}

Patients with gastrointestinal symptoms with known invasive lobular breast cancer should be adequately investigated for potential metastases through imaging or gastroscopy and colonoscopy, to rule out presence of gastrointestinal metastases. Particular attention to the subtype of ILC and prognostic factors including age and nodal involvement, should alert clinicians to the likely possibility for gastrointestinal metastases. 
Knowledge of this unusual pattern of disease will help plan appropriate treatment, in order to optimise prognosis.

\section{Acknowledgements}

The authors thank Dr Jonathan A Smiles, Pathologist for his contribution to the paper.

\section{Conflict of interest}

None declared

\section{Ethical approval}

This study does not require any ethical committee approval.

\section{Author contribution}

WY, SK and AF were all involved in reviewing the literature, prepared and edited the manuscript and all authors approved the final version of the manuscript.

\section{References}

1. Molina-Barea R, Rios-Peregrina, R., Slim, M., Calandre, E, P., Hernandez-Garcia, M, D., Jienez-Rios, J, A. 2014. Lobular breast cancer metastasis to the colon, appendix and the gallbladder Breast Care:428-30.

2. Miller T. 2013 Unexpected Diagnosis of Both Adenocarcinoma of the Colon and Metastatic Lobular Carcinoma of the Breast in the Gastrointestinal Tract. Hindawi.

3. He H, Gonzalez, A., Robinson, E., Yang, W, T. 2014. Distant Metastatic Disease Manifestations in Infiltrating Lobular Carcinoma of the Breast. American Journal of Roentgenology;202:1140-8.

4. Santini D, Altomare A, Vincenzi B, et al. 2006. An increase of Ca 19.9 as the first clinical sign of ilocecal valve metastasis from breast cancer In Vivo;20:165-8.

5. Borst M, Ingold JA Metastatic patterns of invasive lobular versus invasive ductal carcinoma of the breast. 1993. Surgery;114:637-41.

6. Ambroggi M, Stroppa, E, M., Mordenti, P., Biasini, C., Zangrandi, A., Michieletti, E., Belloni, E., Cavanna, L. Metastatic Breast Cancer to the Gastrointestinal Tract: Report of Five Cases and Review of the Literature. 2012. International Journal of Breast Cancer. Article ID: 439023

7. Corso G, Pravetooni, G., Galimberti, V., Veronsei, P. Clinical implication of E-cadherin deficiency in lobular breast cancer. 2019. Breast Cancer Research and Treatment;173:751-2.

8. Shah K, Chuang, K., Srinivasan, I. A Rare Case of Lobular Breast Cancer with Metastasis to the colon, 2019. American Journal of Gastroenterology;114:833-4.

9. Ferlicot S, Vincent-Salomon, A., Medioni, J., Genin, P., Rosty, C., Sigal-Zafrani, B., Freneaux, P., Jouuve, M., Thiery, J, P., Sastre-Garau, X. 2004. Wide Metastatic Spreading in Infiltrating Lobular Carcinoma of the Breast. European Journal of Cancer;40:336-41.

10. Younis L, K., Sakka, H, E., Haque, I. The Prognostic Value of E-cadherin Expression in Breast cancer. 2007. International Journal of Health Sciences;1:43-51.

11. Kanthilatha, P., Baliga, P., Shrestha, B. 2013. E-Cadherin Expression: A Diagnostic Utility for Differentiating Breast Carcinomas with Ductal and Lobular Morphologies, Journal of Clinical and Diagnostic Research; 7 (5), 840-844.

12. Lorfida M, Maiorano, E., Orvieto, E., Maisonneuve, P., Bottiglieri, L., Rotmensz, N., Montagna, E., Dellapasqua, S., Veronesi, P., Galimberti, V., Luini, A., Goldhirsch, A., Colleoni, M., Viale, G. Invasive Lobular Breast Cancer: Subtypes and Outcome. 2012. Breast Cancer Research and Treatment;133:713-23. 


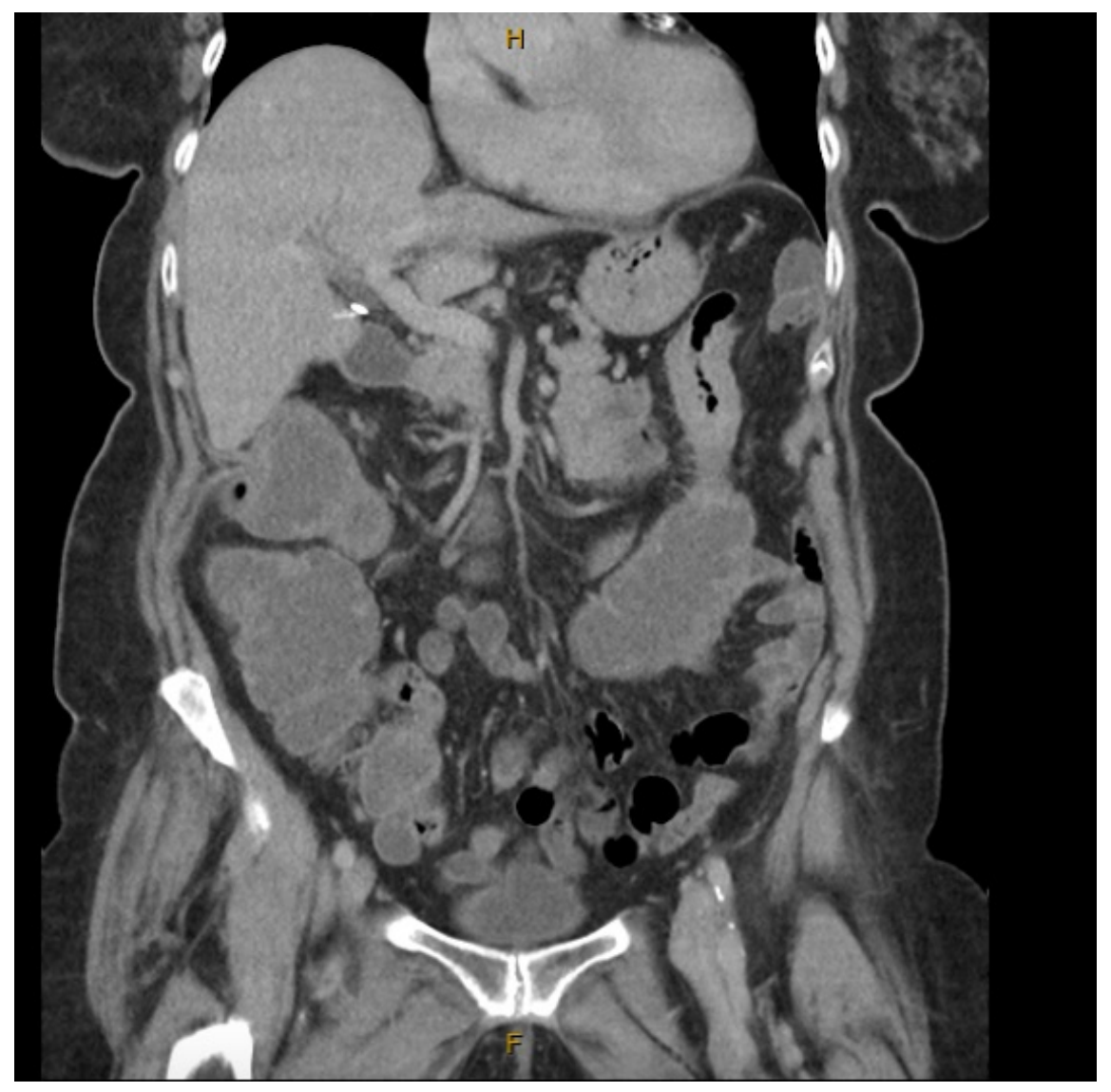




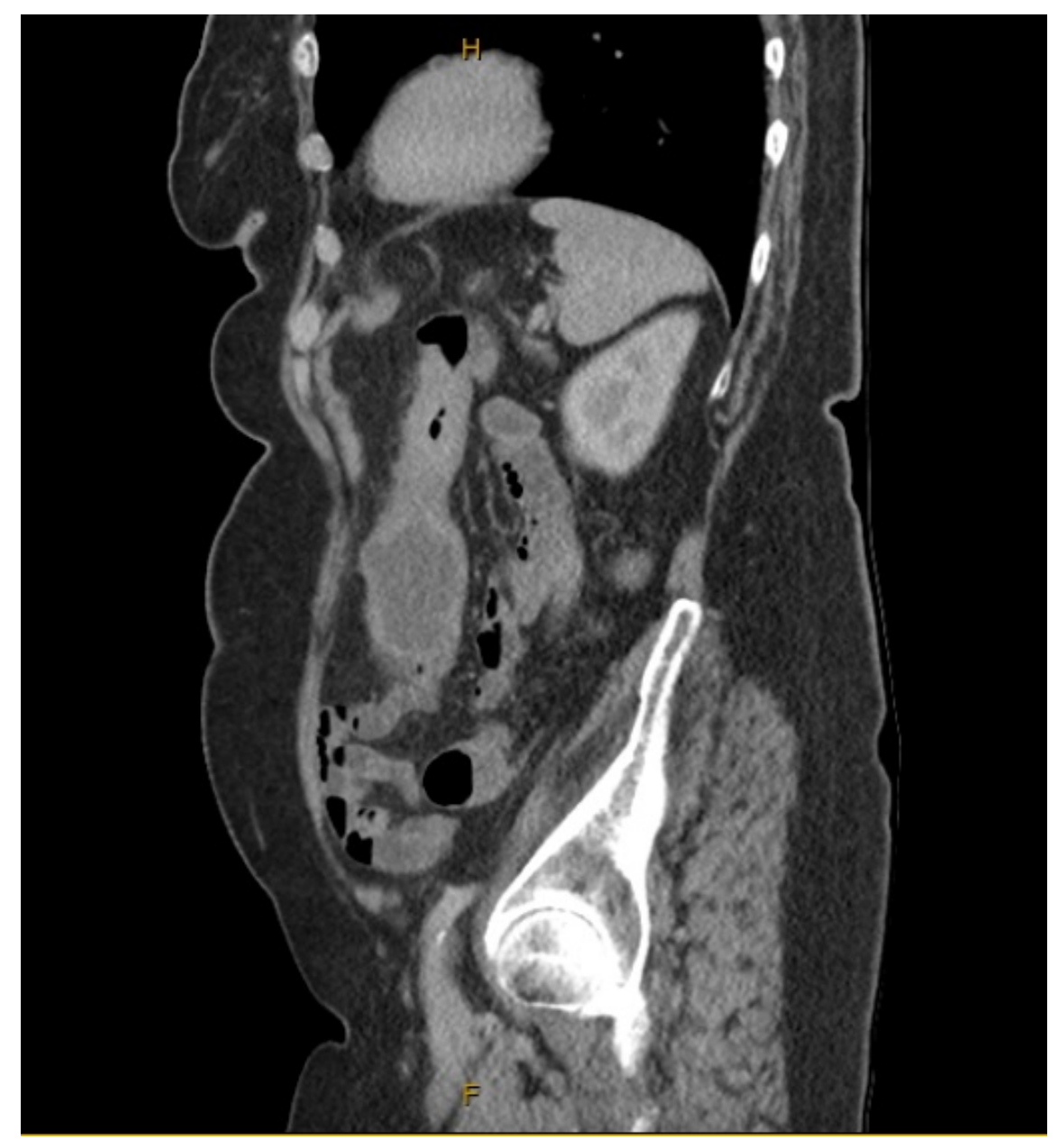




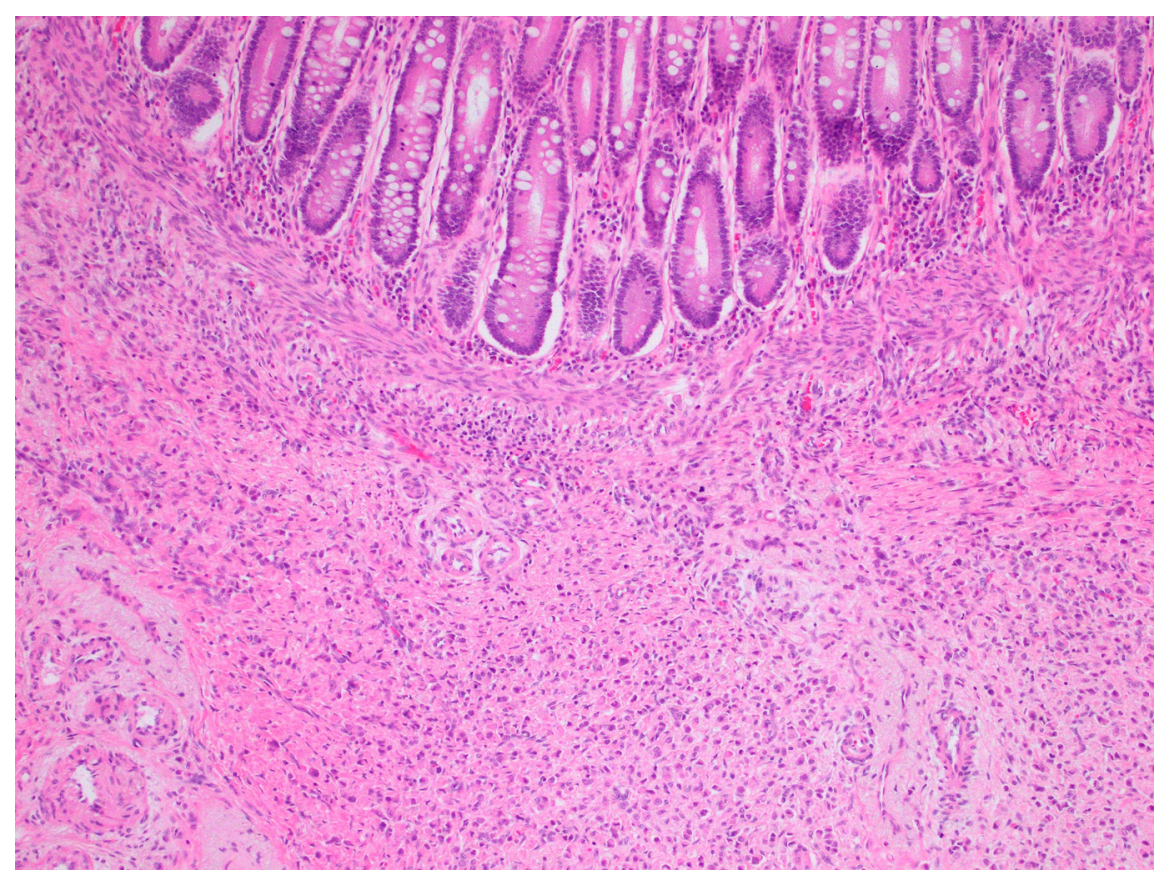

